TRANSACTIONS OF THE

AMERICAN MATHEMATICAL SOCIETY

Volume 354, Number 6, Pages 2173-2185

S 0002-9947(02)02883-0

Article electronically published on February 1, 2002

\title{
COTENSOR PRODUCTS OF MODULES
}

\author{
L. ABRAMS AND C. WEIBEL
}

\begin{abstract}
Let $C$ be a coalgebra over a field $k$ and $A$ its dual algebra. The category of $C$-comodules is equivalent to a category of $A$-modules. We use this to interpret the cotensor product $M \square N$ of two comodules in terms of the appropriate Hochschild cohomology of the $A$-bimodule $M \otimes N$, when $A$ is finite-dimensional, profinite, graded or differential-graded. The main applications are to Galois cohomology, comodules over the Steenrod algebra, and the homology of induced fibrations.
\end{abstract}

\section{INTRODUCTION}

Cotensor product and its derived functors Cotor $^{i}$ were originally introduced by Eilenberg-Moore $[\mathrm{EM}$ to calculate the homology of an induced fibration. These functors have been discussed in the literature, primarily in homotopy theory, but they have developed a reputation of being inaccessible. To counter this, we describe cotensor product and Cotor in terms of Hochschild cohomology, and provide a new class of examples to which they are relevant. Let $C$ be a coalgebra over a field $k$ and $A$ its dual algebra. It is classical [Car that one can identify comodules over $C$ with a subcategory of modules over $A$. Thus comodule constructions may be translated into module constructions. In this paper, we interpret the cotensor product $M \square N$ of two comodules as the vector space $\operatorname{Hom}_{A^{e}}(A, M \otimes N)$ of bimodule maps from $A$ to $M \otimes N$. We also interpret the right derived functors $\operatorname{Cotor}_{C}^{*}(M, N)$ in terms of the Hochschild cohomology of the $A$-bimodule $M \otimes N$. Our results involve several apparently disparate areas of mathematics.

When $C$ and $A$ are finite-dimensional, we do indeed have $\operatorname{Cotor}_{C}^{n}(M, N)=$ $H^{n}(A, M \otimes N)$. This straightforward result is presented in Theorem 3.4

When $C$ is infinite-dimensional, it is important to think of $A$ as a topological algebra, as first suggested by Radford $\mathrm{Rad}$. In this case, we prove in Theorem 4.11 that $\operatorname{Cotor}_{C}^{n}(M, N)$ is the continuous Hochschild cohomology $H_{\text {ctn }}^{n}(A, M \otimes N)$, which we define in 4.6 below.

This result has an interesting application to the Galois cohomology of a profinite group $G$. The profinite group ring $k[G]$ is dual to the coalgebra $C$ of locally constant functions from $G$ to $k$, and the Galois cohomology $H_{\text {ctn }}^{*}(G, M)$ of $G$ with coefficients in a discrete $G$-module $M$ is the same as $\operatorname{Cotor}_{C}^{*}(M, k)$; see Section 4 for details.

When $C$ is graded and each $C_{n}$ is finite-dimensional, we prove that $\operatorname{Cotor}_{C}^{*}(M, N)$ is the graded Hochschild cohomology $H_{g r}^{*}(M, N)$, at least when the graded modules

Received by the editors April 18, 2000 and, in revised form, June 14, 2001.

2000 Mathematics Subject Classification. Primary 16E30; Secondary 16W30, 16E40.

The second author was partially supported by NSF grants.

(C)2002 American Mathematical Society 
$M$ and $N$ are bounded above (Theorem [5.6). Here there is extra bookkeeping for indices, but no topology is needed.

For example, consider the Steenrod algebra $A^{*}$ over $\mathbb{F}_{p}$, and let $X, Y$ denote topological spaces. Their homology groups are comodules over the dual $A_{*}$, and we have $\operatorname{Cotor}_{A_{*}}^{n}\left(H_{*}(X), H_{*}(Y)\right)=H^{n}\left(A^{*}, H_{*}(X \times Y)\right)$; see Section 5 .

In Section 6 we extend our results to the differential graded case, and tie them in with the original formulation and application of Eilenberg and Moore [EM].

The special case when $A$ is a Frobenius algebra (and therefore finite-dimensional) was previously considered by the first author in $\mathrm{Abr}$. The construction there makes use of the special duality characterizing Frobenius algebras to produce a coalgebra structure on the dual of $A$ and to translate the comodule constructions to module constructions. Proposition 3.3 here fills in a gap in the proof of [Abr, 4.6.1].

Notation. The symbol $\otimes$ denotes tensor product over $k$, and the term "algebra" always refers to a $k$-algebra. For any vector space $V$, write $D V$ for the dual space $\operatorname{Hom}_{k}(V, k)$. When $V$ is finite-dimensional there are natural isomorphisms $D\left(V_{1} \otimes V_{2}\right) \cong\left(D V_{1}\right) \otimes\left(D V_{2}\right)$ and $V \cong D(D V)$.

For a $k$-algebra $A, A^{e}$ denotes the enveloping algebra $A \otimes A^{\text {op }}$. Thus a map $B_{1} \rightarrow B_{2}$ of $A-A$ bimodules may be described as a map of left $A^{e}$-modules. We write $\operatorname{Hom}_{A^{e}}\left(B_{1}, B_{2}\right)$ for the set of such maps.

\section{Modules ANd COMOdules}

The results in this section are well known; they appear in $\overline{\mathrm{Car}}$ and later on in $\underline{\mathrm{Sw}}$. Let $k$ be a field and $A$ a finite-dimensional algebra. It is elementary (see $\underline{\mathrm{Sw}}$ 1.1.2]) that the dual of the structure maps $k \rightarrow A$ and $A \otimes A \rightarrow A$ make the dual $D A=\operatorname{Hom}_{k}(A, k)$ into a co-associative coalgebra.

If $M$ is a left $A$-module, the formula $\Delta_{M}(m)(a)=a m$ defines a map

$$
\Delta_{M}: M \rightarrow \operatorname{Hom}_{k}(A, M)=D A \otimes M \cong M \otimes D A .
$$

By $[\mathrm{Car}, 4-03]$ or $[\mathrm{Sw}, 2.1 .2]$, this makes $M$ into a right $D A$-comodule. Conversely, if $M$ is a right $D A$-comodule the map

$$
A \otimes M \stackrel{1 \otimes \Delta}{\longrightarrow} A \otimes(M \otimes D A) \cong(A \otimes D A) \otimes M \stackrel{e v \otimes 1}{\longrightarrow} M
$$

makes $M$ into a left $A$-module by $[\mathrm{Sw}, 2.1 .1]$. As observed in loc. cit., these processes are inverse to each other, and module maps correspond to comodule maps by Car. or $[\mathrm{Sw}, 2.1 .3(\mathrm{e})]$. We summarize this as follows.

Theorem 2.1. Let $A$ be a finite-dimensional algebra. Then there is an equivalence between the category of left A-modules and the category of right DA-comodules.

$$
A-\bmod \cong \operatorname{comod}-D A
$$

Note in particular that the comodule structure map

$$
\Delta_{A}: A \rightarrow \operatorname{Hom}_{k}(A, A) \cong \operatorname{End}_{k}(A)
$$

is the right regular representation: $\Delta_{A}(b)(a)=a b$. In particular, the composition with $k \rightarrow A$ yields the usual trace map $\tau_{A}: k \rightarrow A \otimes D A \cong \operatorname{End}_{k}(A)$.

When $M$ is finite-dimensional, we can use duality to give another interpretation of this equivalence. If $M$ is any left $A$-module, $\operatorname{Hom}_{k}(M, k)$ is naturally a right $A$-module by the rule $(f a)(m)=f(a m)$. If $M$ is finite-dimensional, the dual of the module structure map $\rho: D M \otimes A \rightarrow D M$ is a map $D \rho: M \rightarrow M \otimes D A$; 
it is straightforward that $D \rho$ makes $M$ into a right $D A$-comodule. Elementary considerations show that this duality $M \mapsto D M$ gives an equivalence between the category of finite-dimensional left $A$-modules and the category of finite-dimensional right $D A$-comodules.

Lemma 2.2. When $M$ is finite-dimensional, the structure map $\Delta_{M}$ agrees with the duality map $D \rho: M \rightarrow M \otimes D A$.

Proof. Let $\left\{e_{i}\right\}$ be a basis for $A$ and $\left\{e^{i}\right\}$ the dual basis of $D A$. By inspection, when $M=A$ we have

$$
(D \rho)(1)\left(e^{i} \otimes e_{j}\right)=\rho\left(e^{i} \otimes e_{j}\right)(1)=\delta_{i j},
$$

so $(D \rho)(1)=\sum e_{i} \otimes e^{i}=\Delta_{A}(1)$. Now given $m \in M$, let $f_{m}: A \rightarrow M$ be the module homomorphism with $f_{m}(1)=m$. By naturality of $\rho$ and $\Delta$,

$$
\Delta_{M}(m)=\left(f_{m} \otimes D A\right) \Delta_{A}(1)=\left(f_{m} \otimes D A\right)(D \rho)(1)=D \rho(m) .
$$

Note that even when $M$ is not finite-dimensional, and hence $D \rho$ might not be a comodule structure map, the map $\Delta_{M}$ can still be expressed by $\Delta_{M}(m)=$ $\left(f_{m} \otimes D A\right)(D \rho)(1)$, where $f_{m}: A \rightarrow M$ is as in the proof of the lemma.

Any construction on $A$-modules translates into a corresponding construction on $D A$-comodules. The contragradient representation is one example.

Definition 2.3. Let $C$ be a finite-dimensional coalgebra. If $M$ is a right $C$ comodule, $D M=\operatorname{Hom}_{k}(M, k)$ is the left $C$-comodule with structure map

$$
\Delta=\Delta_{D M}: D M \rightarrow D M \otimes C \cong \operatorname{Hom}(M, C)
$$

defined by the formula $(\Delta f)(m)=(f \otimes 1)(\Delta m)$.

We leave it to the reader to check that this is the comodule structure obtained by regarding $M$ as a left module over $A=D C$, and translating the right $A$-module structure on $D M$ into a left $D A$-comodule structure.

Remark 2.4. As an exercise, the reader might enjoy verifying that $\Delta_{D M}$ is the dual of the map $\mu_{M}: A \otimes M \rightarrow M$.

\section{Cotensor product And Cotor}

In this section, $A$ will be a finite-dimensional $k$-algebra. If $M$ is a left $A$-module, and $N$ a right $A$-module, then the results of the last section allow us to view $M$ as a right $D A$-comodule, and $N$ as a left $D A$-comodule. As such, we can form the cotensor product $M \square N=M \square_{D A} N$. Recall from [EM] [Rav] that $M \square N$ is defined to be the kernel of the map

$$
\phi=\phi_{M, N}: M \otimes N \rightarrow M \otimes D A \otimes N, \quad \phi(m \otimes n)=\Delta_{1}(m) \otimes n-m \otimes \Delta_{2}(n) .
$$

Here $\Delta_{1}: M \rightarrow M \otimes D A$ and $\Delta_{2}: N \rightarrow D A \otimes N$ are the comodule structure maps.

Proposition 3.1. If $M$ and $N$ are left and right $A$-modules, respectively, then there is a natural isomorphism

$$
M \square_{D A} N \cong \operatorname{Hom}_{A^{e}}(A, M \otimes N)
$$


Proof. Note first that every bimodule map $f: A \rightarrow M \otimes N$ is determined by $f(1) \in$ $M \otimes N$, so the map $\operatorname{Hom}_{A^{e}}(A, M \otimes N) \rightarrow M \otimes N$ defined by $f \mapsto f(1)$ is injective. To identify the image, consider $\omega=\sum m_{i} \otimes n_{i}$. The assertion that $\omega=f(1)$ for some bimodule map $f$ is equivalent to the condition

$$
\sum a \cdot m_{i} \otimes n_{i}=\sum m_{i} \otimes n_{i} \cdot a, \text { for all } a \in A .
$$

Now, (目) holds if and only if, for all $a \in A$,

$$
\left(1_{M} \otimes a \otimes 1_{N}\right)\left(\sum \Delta_{1}\left(m_{i}\right) \otimes n_{i}\right)=\left(1_{M} \otimes a \otimes 1_{N}\right)\left(\sum m_{i} \otimes \Delta_{2}\left(n_{i}\right)\right),
$$

or, equivalently, if and only if

$$
\sum \Delta_{1}\left(m_{i}\right) \otimes n_{i}=\sum m_{i} \otimes \Delta_{2}\left(n_{i}\right)
$$

i.e., if and only if $\omega \in M \square N$. Thus $f(1) \in M \square N$ for all bimodule maps $f$, and each $\omega \in M \square N$ defines a bimodule map $f$ with $f(1)=\omega$.

Remark 3.2. Because Hochschild cohomology is defined as a functor on bimodules (see $\S \S 4$ and 5 ), it is useful to generalize the $\square$ construction to $A$-bimodules. If $B$ is an $A$-bimodule, the left and right $A$-module structures yield maps $\Delta_{1}: B \rightarrow B \otimes D A$ and $\Delta_{2}: B \rightarrow D A \otimes B$. Writing $T$ for the twisting operator, we set

$$
\phi_{B}=\Delta_{1}-T \Delta_{2}: B \rightarrow B \otimes D A,
$$

and define $\square(B):=\operatorname{ker}\left(\phi_{B}\right)$. If $B=M \otimes N$, where $M$ and $N$ are left and right $A$-modules respectively, then this $\phi_{B}$ agrees with the previous $\phi_{M, N}$ modulo the natural twist isomorphism $M \otimes D A \otimes N \cong M \otimes N \otimes D A$. By definition, $\square(M \otimes N)=$ $M \square N$.

Choose a $k$-basis $\left\{e_{i}\right\}$ for $A$ and dual basis $\left\{e^{i}\right\}$ for $D A$. In the particular case $B=A \otimes A$, the structure maps $\Delta_{1}, \Delta_{2}$ are essentially the right and left regular representations, respectively. In terms of the bases we have, for any $b \in B=A \otimes A$,

$$
\phi_{B}(b)=\sum_{i, j}\left(e_{i} b \otimes e^{i}-b e_{j} \otimes e^{j}\right)=\sum_{i}\left(e_{i} b-b e_{i}\right) \otimes e^{i}
$$

By naturality of $\phi$ we can use a bimodule map $A \otimes A \rightarrow B$ to show that this formula holds for any bimodule $B$.

To describe the right derived functors of $\square$, we require a preliminary result on injective modules over finite-dimensional algebras.

Proposition 3.3. If $E_{1}$ is an injective left $A$-module, and $E_{2}$ is an injective right $A$-module, then $E_{1} \otimes E_{2}$ is an injective left $A^{e}$-module.

Proof. Because $A$ is noetherian, every injective module is a direct sum of indecomposable modules $[\mathrm{AF}, 25.6]$. Since direct sums of injective $A^{e}$-modules are injective [AF, 18.13], we may assume that $E_{1}$ and $E_{2}$ are indecomposable injectives, i.e., $E_{1}=E\left(A / I_{1}\right)$ and $E_{2}=E\left(A / I_{2}\right)$ for some one-sided ideals $I_{1}$ and $I_{2}$ (see [AF, 18.12.3]). But there are surjections $A^{n} \rightarrow D\left(A / I_{i}\right)$, yielding injections $A / I_{i} \rightarrow(D A)^{n}$. Hence $E_{1}$ and $E_{2}$ are direct summands of some $(D A)^{n}$. A fortiori, $E_{1} \otimes E_{2}$ is a direct summand of $(D A)^{n} \otimes(D A)^{n}$, which is a sum of copies of the injective $A^{e}$-module $D A \otimes D A$.

A hint to the following result appears in [Doi, 3.1], but the discussion there remains in the category of comodules. 
Theorem 3.4. Let $A$ be a finite-dimensional algebra. If $M$ is a left $A$-module, and $N$ a right $A$-module, there is a natural isomorphism

$$
\operatorname{Cotor}_{D A}^{*}(M, N) \cong H^{*}(A, M \otimes N) .
$$

Proof. This is a formal consequence of 3.1 and 3.3 Choosing an injective resolution $M \rightarrow E_{1}^{*}$ in $A$-mod, and $N \rightarrow E_{2}^{*}$ in $\bmod -A$, the Cotor groups are the cohomology of the chain complex

$$
E_{1}^{*} \square E_{2}^{*} \cong \operatorname{Hom}_{A^{e}}\left(A, E_{1}^{*} \otimes_{k} E_{2}^{*}\right) .
$$

On the other hand, $M \otimes N \rightarrow E_{1}^{*} \otimes E_{2}^{*}$ is an injective resolution in $A^{e}$-mod, so by 3.3 the same complex computes the Hochschild cohomology groups $H^{*}(A, M \otimes N)=$ $\operatorname{Ext}_{A^{e}}^{*}(A, M \otimes N)$.

Classically, the Hochschild cohomology of $M \otimes N$ is given by a standard cochain complex (arising from the bar construction [Wei, 9.1.1]) whose $n$th term is $\operatorname{Hom}_{k}\left(A^{\otimes n}, M \otimes N\right)$. On the other hand, the Cotor groups over $C=D A$ are given by a standard cochain complex (arising from the cobar construction [Rav, A1.2.4]) whose $n$th term is $M \otimes C^{\otimes n} \otimes N$. We leave it as an exercise for the reader to show that these complexes are isomorphic, using the isomorphisms $(D A)^{\otimes n} \otimes V \cong$ $\operatorname{Hom}_{k}\left(A^{\otimes n}, V\right)$.

Example 3.5. As an application, note that $\operatorname{Cotor}_{C}^{1}(M, N)$ is the homology of $M \otimes N \rightarrow M \otimes C \otimes N \rightarrow M \otimes C \otimes C \otimes N$. We can interpret an element of the middle group as a $k$-linear map $f: A \rightarrow M \otimes N$. The kernel of the second map consists of the crossed homomorphisms, and the first map sends $m \otimes n$ to the principal derivation $f$ given by $f(a)=a m \otimes n-m \otimes n a$. This shows directly that $\operatorname{Cotor}_{C}^{1}(M, N)$ is identified with the classical description of $H^{1}(A, M \otimes N)$ as the group of crossed homomorphisms on $M \otimes N$, modulo the subgroup of principal derivations; see [Wei 9.2.1].

Similarly, elements of $\operatorname{Cotor}_{C}^{2}(M, N)$ correspond to coalgebra extensions $C \subset E$ with $E=C \wedge C[\mathrm{Sw}, 9.0]$ and $E / C \cong D M \otimes D N$ (as a bi-comodule), at least when $M$ and $N$ are finite dimensional. To see this, we merely identify $\operatorname{Cotor}_{C}^{2}(M, N)$ with $H^{2}(A, M \otimes N)$, whose elements are classically in one-to-one correspondence with (square zero) algebra extensions $0 \rightarrow M \otimes N \rightarrow B \rightarrow A \rightarrow 0$, and dualize. See Wei, 9.3.1]. When $M$ and $N$ are infinite dimensional, the dual $E$ of $B$ is properly thought of as an inverse limit of finite extensions $C \subset E_{i}$.

\section{Profinite algebras}

We now generalize the results of $\$ 3$ to comodules over an arbitrary coalgebra $C$. The development here parallels the development of Galois cohomology in Wei. $\S 6.11$ ], and in particular Theorem 4.9] is the analogue of [Wei, 6.11.13].

Given any right $C$-comodule $M$, we can make $M$ into a left module over $A=D C$, via the composite [Car] $\mathrm{Sw}, 2.1 .1]$

$$
A \otimes M \stackrel{1 \otimes \Delta}{\longrightarrow} A \otimes(M \otimes C) \cong M \otimes(A \otimes C) \stackrel{1 \otimes e v}{\longrightarrow} M .
$$

Similarly, we can make any left $C$-comodule $N$ into a right $A$-module. As before, we can consider $M \otimes N$ as an $A$-bimodule. The proof of Proposition 3.1 readily extends to the current context. 
Proposition 4.1. Let $M$ and $N$ be right and left $C$-comodules, respectively. There is a natural isomorphism

$$
M \square_{C} N \cong \operatorname{Hom}_{A^{e}}(A, M \otimes N) .
$$

The analogue of Theorem 3.4 for the Cotor groups requires a more robust module context, which begins with the correspondence between arbitrary coalgebras and profinite algebras.

Any coalgebra $C$ is the union of its finite-dimensional subcoalgebras $C^{\alpha}$, so its dual $A=\operatorname{Hom}_{k}(C, k)$ is the inverse limit of the finite-dimensional algebras $A_{\alpha}=$ $D\left(C^{\alpha}\right)$. We view $A=\left\{A_{\alpha}\right\}$ as a pro-object in the category of finite-dimensional algebras, a structure we will refer to as a profinite algebra. Each $A_{\alpha}$ is isomorphic to $A / I_{\alpha}$, where $I_{\alpha}$ is the ideal of functions vanishing on $C^{\alpha}$; these ideals define a topology on $A$.

Conversely, suppose that $A=\left\{A_{\alpha}\right\}$ is a profinite algebra. Then the union $C$ of the filtered system of duals $C^{\alpha}=D\left(A_{\alpha}\right)$ is a coalgebra, and clearly $A$ is the profinite algebra associated to $C$. This establishes an equivalence between the category of coalgebras and the opposite category of profinite algebras $[\mathrm{Br}]$ Wit].

Note that a profinite algebra carries more structure, (e.g. topology) than its inverse limit. This is clearly seen in the papers by Radford and Witkowski on reflexive coalgebras $[\mathrm{Rad}$ Wit].

Definition 4.2. Let $A=\left\{A_{\alpha}\right\}$ be a profinite algebra. We say that a (left) $A$-module $M$ is rational if each element of $M$ generates a finite-dimensional $A$ submodule isomorphic to a quotient of some $A_{\alpha}$.

The category $A$-mod $\mathbf{r a t}$ of rational modules and $A$-module maps is an abelian category, and the inclusion $A$-mod $\mathbf{r a t} \subset A$-mod is exact. The argument of [Wei] 6.11.10] shows that $A$-mod rat $_{\text {ras }}$ enough injectives.

Radford [Rad, 2.2] and Witkowski [Wit] have shown that our notion of rational module agrees with Sweedler's notion $[\mathrm{Sw}$ p. 37]. That is, an $A$-module $M$ is rational in our sense if and only if the map $M \stackrel{\rho}{\rightarrow} \operatorname{Hom}(A, M)$ defined by $\rho(m)(a)=$ $a \cdot m$ lands in the subspace $M \otimes C$ of $\operatorname{Hom}(A, M)$.

If $M$ is a rational module, we can regard it as a $C$-comodule as follows. Since $M=\bigcup A m$, it suffices to consider $A m$. Choose $\alpha$ so that $A m$ is an $A_{\alpha}$-module. By Theorem 2.1, $A m$ is a comodule over $C^{\alpha}$ and hence over $C$. This proves Sweedler's theorem $[\mathrm{Sw}, 2.1 .3]$, which we record here.

Theorem 4.3. If $C$ is a coalgebra and $A$ the dual profinite algebra, then there is an equivalence between the category of right $C$-comodules and the category of left rational A-modules.

$$
A-\text { mod }_{\text {rat }} \cong \operatorname{comod}-C .
$$

Example 4.4. Let $G$ be a profinite group, with finite quotients $G_{\alpha}$. The group rings $k\left[G_{\alpha}\right]$ are dual to the Hopf algebras $k^{G_{\alpha}}$ of functions $G_{\alpha} \rightarrow k$. Hence the profinite group algebra $\left\{k\left[G_{\alpha}\right]\right\}$ corresponds to the coalgebra $C=\bigcup k^{G_{\alpha}}$ of locally constant functions $G \rightarrow k$. Regarding each $G_{\alpha}$ as the algebraic group $\operatorname{Spec}\left(k^{G_{\alpha}}\right)$, we get a pro-algebraic group $\left\{G_{\alpha}\right\}$.

A rational $k[G]$-module is the same thing as a discrete $G$-module in the sense of Galois cohomology [Wei]. By Sweedler's theorem, it is also just a comodule for $C$. Now a rational representation of the pro-algebraic group $\left\{G_{\alpha}\right\}$ is just a union of rational representations of the $G_{\alpha}$, each of which is just a $k^{G_{\alpha}}$-comodule (see Fog 
$2.23])$. Thus a discrete $G$-module may be thought of as a rational representation of $\left\{G_{\alpha}\right\}$.

Example 4.5. (Taft [T]) An $A$-module $M$ is called locally finite if each element of $M$ generates a finite-dimensional $A$-submodule. This notion does not involve the topology on $A$, and is weaker than the notion of rational module.

For example, let $V$ be an infinite-dimensional $k$-vector space and form the coalgebra $C:=k \oplus V$, where the elements of $V$ are primitive. The dual algebra is $A=k \oplus V^{*}$. The $A$-module $M:=V^{* *}$ is locally finite (for each $m \in M$, the submodule $A m$ is two-dimensional) but not rational ( $A m$ need not be a quotient of any $A_{\alpha}$ ).

We now turn to $A$-bimodules. In order to define continuous Hochschild cohomology, we again need to consider the topology of $A$.

Definition 4.6. We say that an $A$-bimodule $B$ is rational if each element of $B$ generates a finite-dimensional sub-bimodule isomorphic to a quotient of some $A_{\alpha}$. That is, $B$ is a rational module over the profinite algebra $A \widehat{\otimes} A^{\mathrm{op}}=\left\{A_{\alpha} \otimes A_{\alpha}^{\mathrm{op}}\right\}$.

We define the continuous Hochschild cohomology of $B$ to be the right-derived functors $H_{\mathrm{ctn}}^{*}(A, B)$ of

$$
B \mapsto \operatorname{Hom}_{A^{e}}(A, B)=\{b \in B: a b=b a \text { for all } a \in A\} .
$$

Recall [Wei, 6.5.1] that if $B$ is an $A$-bimodule, its Hochschild cohomology $H^{*}(A, B)$ is defined to be the homology of the cochain complex $C^{*}(A, B)$, where $C^{n}(A, B)$ denotes the $k$-module of $(n+1)$-fold multilinear maps from $A$ to $B$, i.e., maps $f: A^{\otimes n} \rightarrow B$. When $n=0, C^{0}(A, B)=B$.

When $A=\left\{A_{\alpha}\right\}$ is a profinite algebra and $B$ is a rational bimodule, we define $C_{\mathrm{ctn}}^{n}(A, B)$ to be the subspace of $C^{n}(A, B)$ consisting of continuous cochains, i.e., maps which factor through some quotient $A_{\alpha}^{\otimes n}$ of $A^{\otimes n}$.

For each finite quotient $A_{\alpha}=A / I_{\alpha}$ of $A$, set $B^{\alpha}:=\left\{b \in B: I_{\alpha} b=b I_{\alpha}=0\right\}$. Then $B^{\alpha}$ is an $A_{\alpha}$-bimodule, and $B=\bigcup B^{\alpha}$ if $B$ is rational.

Proposition 4.7. We have $C_{\mathrm{ctn}}^{*}(A, B)=\underline{\lim } C^{*}\left(A_{\alpha}, B^{\alpha}\right)$.

Proof. The composite of $A^{\otimes n} \rightarrow A_{\alpha}^{\otimes n}$ with a map $A_{\alpha}^{\otimes n} \rightarrow B^{\alpha}$ is a continuous cochain, so $C^{*}\left(A_{\alpha}, B^{\alpha}\right) \rightarrow C^{*}(A, B)$ is an injection. Conversely, given a continuous cochain $f: A^{\otimes n} \rightarrow B$, there is an $\alpha$ so that $f$ factors through $A_{\alpha}^{\otimes n}$. Because $A_{\alpha}^{\otimes n}$ is finite-dimensional and $B$ is rational, the image $f\left(A^{\otimes n}\right)$ lies in some $B^{\beta}$. Choosing $\gamma$ so that $A_{\gamma}$ maps to $A_{\alpha}$ and $A_{\beta}$, we see that $f \in C^{n}\left(A_{\gamma}, B^{\gamma}\right)$.

Remark 4.8. Our terminology comes from the fact that the profinite algebra $A \widehat{\otimes} \ldots$ $\widehat{\otimes} A=\left\{A_{\alpha}^{\otimes n}\right\}$ may be regarded as a topological algebra. If $B$ has the discrete topology, a continuous map $A \widehat{\otimes} \cdots \widehat{\otimes} A \rightarrow B$ must factor through some $A_{\alpha}^{\otimes n}$ and hence be a continuous cochain.

Theorem 4.9. If $B$ is a rational bimodule over a profinite algebra $A$, then $H_{\mathrm{ctn}}^{*}(A, B)$ is the cohomology of the complex $C_{\mathrm{ctn}}^{*}(A, B)$. In particular,

$$
H_{\mathrm{ctn}}^{*}(A, B) \cong \lim _{\longrightarrow} H^{*}\left(A_{\alpha}, B^{\alpha}\right) .
$$

Proof. For simplicity, set $T^{n}(B)=H^{n} C^{*}(A, B)=\underline{\lim } H^{n} C^{*}\left(A_{\alpha}, B^{\alpha}\right)$. Note that $C^{0}(A, B) \cong B$, and thus $T^{0}(B)=H_{\mathrm{ctn}}^{0}(A, B)$. We now argue as in the proof of Wei] 
6.11.13]. The set $\left\{T^{n}\right\}$ forms a $\delta$-functor because $H^{*} C^{*}\left(A_{\alpha}, B^{\alpha}\right)$ is the Hochschild cohomology $H^{*}\left(A_{\alpha}, B^{\alpha}\right)$ for each $\alpha$. To see that this $\delta$-functor is universal, note that if $J$ is an injective object in $A \widehat{\otimes} A-\bmod _{\text {rat }}$, then each $J^{\alpha}$ is an injective $A_{\alpha^{-}}$ bimodule (because $B \mapsto B^{\alpha}$ is right adjoint to the forgetful functor). Hence if $n \neq 0$ then

$$
T^{n}(J)=\underset{\lim }{\longrightarrow} H^{n}\left(A_{\alpha}, J^{\alpha}\right)=0
$$

Example 4.10. Let $G=\left\{G_{\alpha}\right\}$ be a profinite group and $M$ a discrete left $G$ module. A continuous cochain $f: G^{n} \rightarrow M$ in the sense of Galois cohomology is one which factors through some quotient $G_{\alpha}^{n}$ of $G^{n}$. Its linear extension $k[G]^{\otimes n} \rightarrow M_{\epsilon}$ is a continuous cochain, where $M_{\epsilon}$ is the bimodule with trivial right $G$-action. Thus $C_{\text {ctn }}^{*}\left(k[G], M_{\epsilon}\right)$ is the chain complex used to compute the Galois cohomology of $G$. It follows that $H_{\mathrm{ctn}}^{*}(k[G], M) \cong H_{\mathrm{ctn}}^{*}(G, M)$.

We can now prove the main theorem.

Theorem 4.11. If $C$ is a coalgebra, $A$ its profinite dual, and $M, N$ are $C$-comodules, then

$$
\operatorname{Cotor}_{C}^{*}(M, N)=H_{\mathrm{ctn}}^{*}(A, M \otimes N) .
$$

Proof. Let $\left\{C^{\alpha}\right\}$ and $\left\{A_{\alpha}\right\}$ be as in the beginning of this section. Define $M^{\alpha}:=$ $\left\{x \in M \mid I_{\alpha} x=0\right\}=\left\{x \in M \mid \Delta_{M} x \in M \otimes C^{\alpha}\right\}$ and define $N^{\alpha}$ similarly. Note that $M^{\alpha} \otimes N^{\alpha}=(M \otimes N)^{\alpha}$. By Theorem $4.3 M, N, M^{\alpha}, N^{\alpha}$ may all be viewed as rational $A$-modules. Note that $\operatorname{Cotor}_{C^{\alpha}}\left(M^{\alpha}, N^{\alpha}\right)$ can be calculated with the cobar complex

$$
M^{\alpha} \otimes N^{\alpha} \rightarrow M^{\alpha} \otimes C^{\alpha} \otimes N^{\alpha} \rightarrow M^{\alpha} \otimes C^{\alpha} \otimes C^{\alpha} \otimes N^{\alpha} \rightarrow \cdots
$$

Taking direct limits, we get a cobar complex for $\operatorname{Cotor}_{C}(M, N)$. Since homology commutes with this particular limit, we have

$$
\operatorname{Cotor}_{C}^{*}(M, N)=\underline{\lim } \operatorname{Cotor}_{C^{\alpha}}^{*}\left(M^{\alpha}, N^{\alpha}\right)
$$

Applying Proposition 4.7 and Theorem 3.4 gives

$$
\underset{\lim }{\longrightarrow} \operatorname{Cotor}_{C^{\alpha}}^{*}\left(M^{\alpha}, N^{\alpha}\right)=\underline{\lim } H^{*}\left(A_{\alpha}, M^{\alpha} \otimes N^{\alpha}\right)=H_{\mathrm{ctn}}^{*}(A ; M \otimes N),
$$

which completes the proof.

Example 4.12. As in Example 3.5, we can interpret a 1-cocycle in the cobar complex for $\operatorname{Cotor}_{C}^{*}(M, N)$ as a continuous map $A \rightarrow M \otimes N$. This leads to the description of $\operatorname{Cotor}_{C}^{1}(M, N)$ as continuous crossed homomorphisms (on $A$ ) modulo principal derivations.

Similarly, if both $M$ and $N$ are finite-dimensional, then $\operatorname{Cotor}_{C}^{2}(M, N)$ classifies coalgebra extensions $C \subset E$ with $E=C \wedge C$ and $E / C \cong D M \otimes D N$ (as a bicomodule). Indeed, this corresponds under duality with the fact that (square zero) profinite-dimensional extensions of $A$ by the bimodule $M \otimes N$ are classified by $H_{\mathrm{ctn}}^{2}(A, M \otimes N)$. 


\section{Graded modules AND COMOdules}

Replacing the category of vector spaces with the category of graded vector spaces does not change things very much, as we now explain.

First, we need to fix our notation, for which we follow $[\mathrm{Mac}]$ and $[\mathrm{EM}]$. The category of graded vector spaces has an internal Hom, constructed as follows. Given two graded vector spaces $V$ and $V^{\prime}$, the degree $p$ component $\operatorname{Hom}_{k}^{p}\left(V, V^{\prime}\right)$ of $\operatorname{Hom}_{k}^{*}\left(V, V^{\prime}\right)$ is the vector space of all maps $f: V \rightarrow V^{\prime}$ of degree $p$, i.e., satisfying $f\left(V^{i}\right) \subset\left(V^{\prime}\right)^{i+p}$. The indexing is set up so that the evaluation map $\operatorname{Hom}_{k}^{*}\left(V, V^{\prime}\right) \otimes$ $V \rightarrow V^{\prime}$ is homogeneous of degree zero. The graded dual $D V=\operatorname{Hom}^{*}(V, k)$ of $V$ arises as the special case $V^{\prime}=k$. Thus $(D V)_{n}=(D V)^{-n}=\operatorname{Hom}_{k}\left(V^{n}, k\right)$. If each $V^{n}$ is finite-dimensional we say that $V$ is of finite type; this is the hypothesis needed to have $D(D V) \cong V$.

By a graded algebra $A$ we will mean a positively graded algebra $A^{0} \oplus A^{1} \oplus \cdots$. We will assume throughout this section that $A$ is of finite type.

By a graded coalgebra $C$ we will mean a positively graded coalgebra $C_{0} \oplus C_{1} \oplus \cdots$. If $A$ is a graded $k$-algebra of finite type, then $D A$ is a co-associative graded coalgebra by [MM, 3.1(4)] or $[\mathrm{Sw}, 6.0 .2]$. This is because the tensor product $A \otimes A$ is of finite type.

Note that a graded module $M$ is rational if and only if it is locally finite (Example 4.5); the topology on $A$ plays no role when $A$ is of finite type. As a typical example, if $M$ is a bounded-above module (i.e., $M^{p}=0$ for $p \gg 0$ ) then clearly $M$ is locally finite, because $A$ has finite type.

Proposition 5.1. If $A$ is of finite type, then the category of graded right DAcomodules is equivalent to the category of locally finite, graded left A-modules. Under this equivalence, the category of bounded-below comodules $\left(M_{p}=0\right.$ for $\left.p \ll 0\right)$ is equivalent to the category of bounded-above modules.

Proof. If $M_{*}$ is a comodule, the classical formula for the $A$-module structure on $M$ (in section 2) makes it a locally finite graded module (see [MM]). Conversely, if $M^{*}$ is a graded module, the formula $\Delta_{M}(m)(a)=a m$ is homogenous, and $\Delta_{M}(m)$ is in $M \otimes D A$ if $M$ is locally finite. Note that $M$ and $D A$ are interchanged exactly once in each of these formulas, so this equivalence is independent of graded sign convention.

Suppose now that $M$ is a graded right comodule and $N$ a graded left comodule. Then we can consider $M$ and $N$ as left and right modules, respectively, and form the graded bimodule $M \otimes N$. Applying the graded $\operatorname{Hom}_{A^{e}}(A,-)$ (defined as in Mac p. 185]) allows us to construct the graded Hochschild cohomology $H_{g r}^{*}(A, M \otimes N)$, as in Mac, p. 300].

The proof of Proposition 3.1 goes through to prove the following analogue.

Proposition 5.2. Let $A$ be a graded algebra of finite type, with dual coalgebra $C$. If $M$ and $N$ are locally finite left and right graded $A$-modules, respectively, then there is a natural isomorphism

$$
M \square_{C} N \cong \operatorname{Hom}_{A^{e}}(A, M \otimes N) .
$$

In order to prove the analogue of Theorem [3.4 we require some preliminary results. 
Lemma 5.3. If $V$ is a bounded above graded vector space, then $D A \otimes V$ is an injective graded $A$-module (and hence also an injective graded $D A$-comodule), and $D A \otimes V \otimes D A$ is an injective graded A-bimodule.

Proof. Because $\operatorname{Hom}_{k}(A,-)$ is right adjoint to the forgetful functor from graded $A$-modules to graded vector spaces, it preserves injectives [Weil 2.3.10]. Hence $\operatorname{Hom}_{k}(A, V)$ is injective. In general, $\operatorname{Hom}_{k}^{n}(A, V)=\prod_{i+j=n} D A^{i} \otimes V^{j}$ is larger than $(D A \otimes V)^{n}=\bigoplus D A^{i} \otimes V^{j}$. But if $V$ is bounded above, we have $D A \otimes V=$ $\operatorname{Hom}(A, V)$, which is injective, and similarly

$$
D A \otimes V \otimes D A=D(A \otimes A) \otimes V=\operatorname{Hom}_{k}\left(A^{e}, V\right)
$$

is injective as a graded $A^{e}$-module, i.e., a graded bimodule.

Remark 5.4. Although $V$ being bounded above is sufficient to guarantee that $D A \otimes$ $V$ is injective, it is not a necessary condition. In particular, if $A$ is a Noetherian algebra, then direct sums of injective modules are injective. Since $D A$ and $D A \otimes$ $D A$ are injective (compare with the proof of Proposition 3.3), we may remove the hypothesis that $V$ be bounded above.

Corollary 5.5. If $M$ and $N$ are bounded-above graded A-modules, there are bounded-above graded vector spaces $V^{i}$ and $W^{i}$ and injective resolutions $M \rightarrow$ $I^{*}, N \rightarrow J^{*}$ with $I^{i} \cong D A \otimes V^{i}, J^{i} \cong W^{j} \otimes D A$. Moreover, $M \otimes N \rightarrow I^{*} \otimes J^{*}$ is an injective bimodule resolution.

Theorem 5.6. Suppose $A$ is a graded k-algebra of finite type, with dual coalgebra $C$, and that $M, N$ are bounded-above graded $A$-modules. Then for each $n$ there is a natural isomorphism of graded vector spaces:

$$
\operatorname{Cotor}_{C}^{n}(M, N) \cong H_{g r}^{n}(A, M \otimes N) .
$$

Proof. Take injective resolutions $M \rightarrow I^{*}$ and $N \rightarrow J^{*}$ as in Corollary 5.5. These lie in the category of graded $D A$-comodules. Because $I^{p}$ and $J^{q}$ are bounded below, the proof of Proposition 3.1 shows that $I^{p} \square J^{q}=\operatorname{Hom}_{A^{e}}\left(A, I^{p} \otimes J^{q}\right)$. Thus we have

$$
\begin{aligned}
\operatorname{Cotor}_{C}^{n}(M, N) & =H^{n}\left(I^{*} \square J^{*}\right) \\
& =H^{n} \operatorname{Hom}_{A^{e}}\left(A, I^{*} \otimes J^{*}\right) \\
& =H_{g r}^{n}(A, M \otimes N) .
\end{aligned}
$$

Remark 5.7. Eilenberg and Moore actually use homological indexing, defining $\operatorname{Cotor}_{n}^{C}(M, N)$ to be the product (over $p, q$ with $p+q=n$ ) of the $\operatorname{Cotor}_{p, q}(M, N)$, which in our notation is the homological degree $p$ part of the graded vector space $\operatorname{Cotor}_{C}^{-q}(M, N)$; see [EM] p. 207].

Example 5.8. Let $A^{*}$ denote the Steenrod algebra over $\mathbb{F}_{p}$. If $X$ is a topological space, its cohomology $H^{*}(X)$ is a bounded-below module over $A^{*}$ and its homology $H_{*}(X)$ is a graded comodule over the dual $A_{*}$. We can view $H_{*}(X)$ as cohomologically bounded above, and hence by $\left[5.1\right.$ as a bounded-above module over $A^{*}$, with $H^{*}(X)=D H_{*}(X)$. Because $H_{*}(X \times Y) \cong H_{*}(X) \otimes H_{*}(Y)$ is a bicomodule over $A^{*}$, we have

$$
H_{*}(X) \square_{A_{*}} H_{*}(Y)=\operatorname{Hom}_{A^{e}}\left(A^{*}, H_{*}(X \times Y)\right) .
$$

and

$$
\operatorname{Cotor}_{A_{*}}^{n}\left(H_{*}(X), H_{*}(Y)\right)=H^{n}\left(A^{*}, H_{*}(X \times Y)\right) .
$$




\section{The Differential Graded Case}

We now pass to the differential graded setting, using essentially the same notation as in the graded case. By a differential graded algebra (or DG-algebra) we will mean a graded algebra $A$ with a differential of degree one, satisfying the usual Leibniz relation for $d\left(a a^{\prime}\right)$. We will regard $A$ as a cochain complex:

$$
0 \rightarrow A^{0} \rightarrow A^{1} \rightarrow \cdots
$$

We use cochain notation $M^{*}$ for DG $A$-modules, so their differential has degree one.

By a differential graded coalgebra (or DG-coalgebra) $C$ we will mean a positively graded coalgebra with a differential of degree -1 , satisfying the co-Leibniz relation. We will regard $C$ as a chain complex:

$$
\cdots \rightarrow C_{1} \rightarrow C_{0} \rightarrow 0 .
$$

For example, $C=D A$ is a DG-coalgebra with $C_{n}=\operatorname{Hom}\left(A^{n}, k\right)$. We use chain complex notation for DG $C$-comodules, so their differential has degree -1 .

Proposition 6.1. If $A$ is of finite type, then the category of right $D G D A$-comodule is equivalent to the category of locally-finite left $D G A$-modules.

Proof. The only point which must be added to the proof for the graded case (Proposition 5.1) is that the same differential is used for both $A$-modules and $D A$ comodules.

If $M$ and $N$ are right and left DG-comodules, then their cotensor product $M \square_{C} N$ is naturally a DG-vector subspace of $M \otimes N$. Yet again, the proof of Proposition 3.1 goes through to prove the following DG analogue.

Proposition 6.2. Let $A$ be a $D G$ algebra of finite type, with dual coalgebra $C$. If $M$ and $N$ are locally finite left and right $D G A$-modules, respectively, then there is a natural isomorphism of $D G$ vector spaces:

$$
M \square_{C} N \cong \operatorname{Hom}_{A^{e}}(A, M \otimes N) .
$$

The cotorsion groups $\operatorname{Cotor}_{n}^{C}(M, N)$ were defined by Eilenberg and Moore in [EM, p. 206] as follows. If $M \rightarrow I^{*}$ and $N \rightarrow J^{*}$ are injective DG-comodule resolutions, then $I^{*} \square_{C} J^{*}$ is a triple chain complex whose $(i, j, k)$ entry is $\left(I^{-i} \square_{C} J^{-j}\right)_{k}$. Here, the index $k$ refers to the total internal grading of the DG-comodules $I^{-i}, J^{-j}$. The group $\operatorname{Cotor}_{n}^{C}(M, N)$ is defined to be the $n$th homology of the (product) total complex of $\left(I^{-i} \square_{C} J^{-j}\right)_{k}$. The homological indexing reflects not only the applications to homology of spaces (see 6.4 below), but the fact that we want $\operatorname{Cotor}_{n}^{C}(C, C) \cong H_{n}(C)$ to be positively indexed.

If $M$ and $N$ are left and right DG $A$-modules, their tensor product $M \otimes N$ may be viewed as a DG-bimodule. As such, we may form the differential graded Hochschild cohomology $H_{D G}^{p}(A, M \otimes N)$.

Theorem 6.3. Suppose $A$ is a DG-algebra of finite type with dual coalgebra $C$ and $M, N$ are bounded-above $D G A$-modules. Then for each $n$ there is a natural isomorphism:

$$
\operatorname{Cotor}_{n}^{C}(M, N) \cong H_{D G}^{-n}(A, M \otimes N) .
$$


Proof. Both Lemma 5.3 and Corollary 5.5 carry over to the DG setting with almost no change. Thus we can choose injective objects $I^{*}$ and $J^{*}$ in the category of DGmodules over $A=D C$ such that $M \otimes N \rightarrow I^{*} \otimes J^{*}$ is an injective resolution in the category of DG-bimodules.

By definition, $H_{D G}^{n}(A, M \otimes N)$ is the $n$th cohomology of the (product) total complex of the triple cochain complex $\operatorname{Hom}_{A^{e}}\left(A, I^{*} \otimes J^{*}\right)$. The $(i, j, k)$ entry of this complex is

$$
\operatorname{Hom}_{A^{e}}\left(A, I^{i} \otimes J^{j}\right)^{k}=\left(I^{i} \square_{C} J^{j}\right)_{-k}
$$

The standard conversion between chain complexes and cochain complexes $\left(V^{n}=\right.$ $V_{-n}$ ) identifies this triple complex with the triple chain complex used above to define $\operatorname{Cotor}^{C}(M, N)$.

Example 6.4. (Eilenberg-Moore) Suppose given a cartesian square of CW complexes

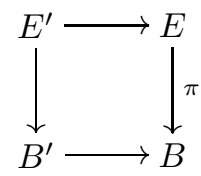

in which $B$ is simply connected, with finitely many cells in each dimension, and $\pi$ is a fibration. Let $C_{*} B$ be the cellular complex of $B$ with coefficients in a field $k$, and $C^{*} B=\operatorname{Hom}\left(C_{*} B, k\right)$ its dual algebra. This is a DG-algebra of finite type. The main result (theorem 12.1) of [EM], combined with our Theorem 6.3 (and the Eilenberg-Zilber theorem) states that the homology of $E^{\prime}$ is isomorphic to

$$
H_{*}\left(E^{\prime} ; k\right) \cong \operatorname{Cotor}^{C * B}\left(C_{*} E, C_{*} B^{\prime}\right) \cong H_{D G}^{*}\left(C^{*} B, C_{*}\left(E \times B^{\prime}\right)\right) .
$$

In particular, if $F$ is the fiber of the fibration $E \rightarrow B$, then (taking $B^{\prime}$ to be a point) we have

$$
H_{*}(F ; k) \cong \operatorname{Cotor}^{C_{*} B}\left(C_{*} E, k\right) \cong H_{D G}^{*}\left(C^{*} B, C_{*} E \otimes k\right) .
$$

Finally, if $E$ is contractible then $C_{*} E \cong k$ and

$$
H_{*}(\Omega B ; k) \cong \operatorname{Cotor}^{C * B}(k, k) \cong H_{D G}^{*}\left(C^{*} B, k\right) \text {. }
$$

\section{REFERENCES}

[Abr] L. Abrams, "Modules, Comodules and Cotensor Product over Frobenius Algebras," J. Algebra 219 (1999), 201-213. MR 2001b:16038

[AF] F. Anderson And K. Fuller, Rings and Categories of Modules, Springer Verlag, 1974. MR 54:5281

[Br] A. Brumer, "Pseudocompact algebras, profinite groups and class formations," J. Algebra 4 (1966), 442-470. MR 34:2650

[Car] P. Cartier, "Cohomology des coalgèbres (Exposés 4,5)," In Séminaire Sophus Lie, 195556, Faculté des Sciences de Paris, 1957.

[CE] H. Cartan and S. Eilenberg, Homological Algebra, Princeton University Press, 1956. MR 17:1040e

[Doi] Y. DoI, "Homological Coalgebra," J. Math Soc. Japan 33, no. 1 (1981), 31-50. MR 82g:16014

[EM] S. Eilenberg And J. Moore, "Homology and fibrations I: Coalgebras, cotensor product and its derived functors," Comm. Math. Helv. 40 (1966), 199-236. MR 34:3579

[Fog] J. FogarTy, Invariant Theory, Benjamin, 1969. MR 39:1458

[Mac] S. MacLane, Homology, Springer Verlag, 1963. MR 28:122 
[MM] J. Milnor And J. Moore, "On the structure of Hopf algebras," Annals of Math. (2) 81 (1965), 211-264. MR 30:4259

[Rad] D. RADFORD, "Coreflexive coalgebras," J. Algebra 26 (1973), 512-535. MR 46:6160

[Rav] D. C. Ravenel, Complex Cobordism and Stable Homotopy Groups of Spheres, Academic Press, 1986. MR 87j:55003

[Sw] M. Sweedler, Hopf Algebras, Benjamin, 1969. MR 40:5705

[T] E. J. TAFT, "Reflexivity of algebras and coalgebras," Amer. J. Math. 94 (1972), 1111-1130. MR 46:9095

[Wei] C. Weibel, An Introduction to Homological Algebra, Cambridge Studies in Adv. Math. 38, Cambridge Univ. Press, 1994. MR 95f:18001

[Wit] L. Witkowski, "On coalgebras and linearly topological rings," Colloquium Mathematicum 40 Fasc. 2 (1979), 207-218. MR 81c:16057

Department of Mathematics, Rutgers University, New Brunswick, New Jersey 08903 Current address: Department of Mathematics, George Washington University, Washington, D.C. 20052

E-mail address: labrams@gwu.edu

Department of Mathematics, Rutgers University, New Brunswick, New Jersey 08903

E-mail address: weibel@math.rutgers.edu 